\title{
High-Speed Photonic Power-Efficient Ultra-Wideband Transceiver Based on Multiple PM-IM Conversions
}

\author{
Enbo Zhou, Xing Xu, Student Member, IEEE, King-Shan Lui, Senior Member, IEEE, and \\ Kenneth Kin-Yip Wong, Member, IEEE
}

\begin{abstract}
We experimentally demonstrate a novel photonic ultra-wideband (UWB) transceiver with pulse spectral efficiency of $50.97 \%$ and transmission speed up to $3.125 \mathrm{~Gb} / \mathrm{s}$. The UWB generator only consists of a highly nonlinear fiber (HNLF) and a commercial arrayed-waveguide grating (AWG). By using the concept of multiple cross-phase modulation in the HNLF and multiple phase modulation to intensity modulation conversions in the AWG, a power-efficient UWB pulse is combined with incoherent summation of two asymmetric monocycle pulses with inverted polarities. Benefiting from the ultra-fast response of fiber nonlinearities in the HNLF, on-off keying encoded UWB signals generated at $781.25 \mathrm{Mb} / \mathrm{s}, 1.5625 \mathrm{~Gb} / \mathrm{s}$, and $3.125 \mathrm{~Gb} / \mathrm{s}$ are all error-free transmitted through a $22.5-\mathrm{km}$ single-mode fiber (SMF) with power penalties lower than $1 \mathrm{~dB}$. The bit-error rate is directly measured on down-converted baseband signals by using optical full rectification and electrical low-pass filtering technologies. The measured electrical spectra before and after $22.5-\mathrm{km}$ SMF link transmission both fully comply with the spectral mask specified by the U.S. Federal Communications Commission (FCC) without power attenuation.
\end{abstract}

Index Terms-Microwave photonics, nonlinear signal processing, ultra-wideband (UWB)-over-fiber, wireless technology.

\section{INTRODUCTION}

I MPULSE radio-based ultra-wideband (UWB) technology with capabilities of high-speed wireless transmission and extremely low radiated power has potential applications in broad areas, e.g., high-speed indoor wireless personal area networks (WPANs), medical industry, sensor networks, and the military. The U.S. Federal Communications Commission (FCC) has approved unlicensed use of the frequency range from 3.1 to $10.6 \mathrm{GHz}$ with radiated signal power density lower than $-41.3 \mathrm{dBm} / \mathrm{MHz}$ in 2002 [1].

The relatively low radiated power density is critical for noninterference operation with other wireless technologies coexisting

Manuscript received January 06, 2010; revised July 28, 2010; accepted July 29, 2010. Date of publication September 30, 2010; date of current version November 12, 2010. This work was supported in part by the Research Grants Council of the Hong Kong Special Administrative Region, China, under Grant Project HKU7179/08E and Grant Project HKU7183/09E.

The authors are with the Photonic Systems Research Laboratory, Department of Electrical and Electronic Engineering, University of Hong Kong, Hong Kong (e-mail: nbzhou@eee.hku.hk; xuxing@eee.hku.hk; kslui@eee.hku.hk; kywong@eee.hku.hk).

Color versions of one or more of the figures in this paper are available online at http://ieeexplore.ieee.org.

Digital Object Identifier 10.1109/TMTT.2010.2074550 in such broad spectral range. Therefore, the FCC has specified a spectral mask to balance the utilization of the sponsors of varies wireless technologies. As a result, a tradeoff between the total radiated power and the power attenuation to comply with the FCC spectral mask emerges. This specification is reflected by its pulse spectral efficiency defined in [2] as the average power of pulse normalized by the total admissible power under the FCC mask within the frequency range of the UWB antenna, generally under $14 \mathrm{GHz}$. As a result, a UWB pulse with high spectral efficiency resulting in high power efficiency without power attenuation to respect the FCC mask is highly desired to effectively utilize the allowable radiated power in the budget. On the other hand, such budget radiated power also limits the operation in the immediate area $(<10 \mathrm{~m})$ lacking in the capability of penetration of walls. To address this shortcoming, UWB-over-fiber technology is proposed to take advantages of low loss in optical fiber-link transmission and the only limitation is the bandwidth of optical-electrical (O/E) conversion [3]. As a result, photonic generation of UWB pulses is considered an effective approach to interconnect the infrastructures of UWB communication systems in optical and electrical domains and has been reported extensively in recent years [2]-[19].

Over the past few years, extensive reports have focused on the photonic generation of lower derivative of Gaussian pulses, e.g., monocycle and doublet pulses [4]-[14]. These approaches can be divided into two categories. Phase modulation to intensity modulation (PM-IM) conversion is a well-known technique that by using a frequency discriminator, either a monocycle or doublet pulse can be recovered if the phase modulated optical carrier is located at either the two oppositely linear slopes or the quadrature slopes of a fiber Bragg grating (FBG) [4]-[7]. Alternatively, via a combination of two Gaussian pulses either by constructively or destructively coherent summation or incoherent summation, UWB pulses can also be obtained [9]-[14]. These low derivative of Gaussian pulses are relatively simple in generation and have center frequencies in the specified range. However, these pulses violate the FCC spectral mask in the low-frequency domain, especially in the notch of the global positioning system (GPS) band (1575 MHz) where a 34-dB power suppression ratio comes into a challenge for all UWB pulses. As a result, power attenuation is required to respect the FCC spectral mask in this frequency range. It is also noted, among these approaches, active-device-based schemes, e.g., semiconductor optical amplifiers (SOAs) and distribution feedback (DFB) lasers, which may be negatively impacted by the 
slow carrier response due to the band-filling effect. As a result, the corresponding spectra have distinct frequency components in the low-frequency domain even in generating a UWB pulse in the form of a high-order derivative of a Gaussian pulse [15], [16]. On the other hand, the highly nonlinear fiber (HNLF) with ultrafast response time inherited from the third-order susceptibility $\left(\chi^{(3)}\right)$ has great potential in generating distortion-free and high-speed UWB pulses [12], [17].

A power-efficient UWB pulse generator based on apodized FBGs is reported recently [2], [18], [19]. By using the apodization technique, arbitrary UWB waveforms can be generated. With optimized design, the generated UWB pulse exploiting spectral efficiency as high as $63.6 \%$ is measured in the experiment. This technique is precise in pulse shaping and efficient in spectrum utilization. However, such an approach with complex experimental setup is at the risk of heavy burden for practical implementation if a balanced receiver end is also included.

In our recent report, we experimentally demonstrated a novel UWB pulse generator based on an HNLF and a commercially available arrayed-waveguide grating (AWG) [21]. Larger than 11.5- and 6-dB improvement on optical signal power compared with monocycle and doublet pulses was achieved, respectively. In this paper, we experimentally demonstrate a high-speed power-efficient UWB transceiver generating a UWB pulse based on the same technique we performed in [21]. FCC-compliant UWB signals are transmitted at three bit rates of $781.25 \mathrm{Mb} / \mathrm{s}, 1.5625 \mathrm{~Gb} / \mathrm{s}$, and $3.125 \mathrm{~Gb} / \mathrm{s}$ following a pattern of a $2^{7}-1$ pseudorandom binary sequence (PRBS) through a $22.5-\mathrm{km}$ single-mode fiber (SMF) link. In the receiver end, the power-efficient UWB signal is directly measured and evaluated in a low speed bit-error rate tester (BERT) after down-conversion to baseband signals by using optical full-rectification and electrical low-pass filtering technologies.

This paper is organized as follows. The principle and theoretical investigation on UWB pulse shaping of our scheme is first introduced in Section II. The experimental setup and the experimental results are given in Section III. In Section IV, we discuss and then conclude this paper.

\section{PRINCIPLE}

\section{A. Design of FCC-Compliant UWB Pulse}

The waveforms having potentials in efficiently exploiting the FCC spectral mask can be expressed by [2]

$$
p(t)=\sum_{n=0}^{L-1} w[n] g\left(t-n T_{0}\right)
$$

where $w[n]$ are $L$ weight coefficients, $T_{0}$ is the pulse spacing, and $g(t)$ is the Gaussian monocycle. It is investigated in [2] that the FCC-compliant UWB pulse without power attenuation requires $L$ at least equal to 7. For lower order terms, $L=2,3$, for instance, the spectral efficiency of the generated monocycle pulses and doublet pulses are relatively low. Note that this investigation is restricted to summation of symmetric monocycles. In the scheme of PM-IM conversion, a transient state leads pulse-shape conversion to a monocycle-like pulse when the optical carrier is located at a linear slope of the transfer function of a detuned filter. Furthermore, if the optical carrier detuned further to either edge of the the linear slope, an asymmetric monocycle pulse with an equal upper and lower pulsewidth, but adjustable amplitude, can be achieved. Such asymmetric monocycle pulses have even undesirable frequency components in low frequency regime. However, by incoherent summation of a pair of polarity opposite asymmetric monocycle pulses with opposite polarity and appropriate time delay, an FCC-compliant UWB pulse with a temporal waveform satisfying (1) for $L=7$ can be achieved.

\section{B. Theory and Simulation}

The previously described generation of an FCC-compliant UWB pulse consists of multiple PM-IM conversions. In our scheme, multiple phase modulation is achieved by XPM in an HNLF, while multiple PM-IM conversions are realized by using a commercially available AWG.

1) Multiple XPM: By pump-probe interaction in an HNLF, the pulsed pump light introduces XPM on probe beams in an HNLF. The pump wavelength avoid being located in the anomalous group-velocity dispersion (GVD) regime near the zero-dispersion wavelength (ZDW) of the HNLF to eliminate cross-gain modulation (XGM) in our scheme [13], [17]. The propagation of the pump and probe light along the fiber transmission direction $z$ is governed by the multiple coupled nonlinear Schrödinger (NLS) equations, given by [22]

$$
\begin{aligned}
\frac{\partial E_{j}}{\partial z}+\frac{1}{v_{g j}} \frac{\partial E_{j}}{\partial t}+ & \frac{i \beta_{2 j}}{2} \frac{\partial^{2} E_{j}}{\partial t^{2}}+\frac{\alpha_{j} E_{j}}{2} \\
& =i\left(\gamma_{j}\left|E_{j}\right|^{2}+2 \sum_{k \neq j} \gamma_{k}\left|E_{k}\right|^{2}\right) E_{j}
\end{aligned}
$$

where $E_{j}$ are the slowly varying electric field envelopes of the pump $(j=1)$ and two probe lights $(j=2,3) . v_{g j}$ are group velocities, $\beta_{2 j}$ are GVD coefficients, $\alpha_{j}$ are the loss coefficients, and $\gamma_{j}$ are the nonlinear parameters of the HNLF of the corresponding components $j$. In our scheme, due to the incoherent summation of two optical sources, two probe lights are required. However, in the simulated results, we find the influence of two probe lights on each other is limited due to this relatively weak power compared with that of the pump light.

2) Multiple PM-IM Conversions: By the principle of wellknown PM-IM conversion, a phase modulated optical carrier locating at the linear slopes of a transfer function of a frequency discriminator leads to PM-IM mapping. In a pulsed pump induced XPM along an HNLF, the pulse leading edge induced red shift and the pulse trailing edge induced blue shift on the probe light are symmetric to each other. As a result, a linear mapping function lead to symmetric derivative of Gaussian pulses, i.e., monocycle pulses. However, if the optical carrier is located at the edges of the linear slopes of the transfer function, asymmetric monocycle pulses are recovered. The waveform recovery can be described by

$$
E_{F, i}(t)=\mathcal{F}^{-1}\left\{H_{\mathrm{AWG}}(\omega) \cdot \mathcal{F}\left[E_{i}(t, L)\right]\right\}
$$

where $i=1,2$ denotes the probe light. $L$ is the length of the HNLF. $H_{\mathrm{AWG}}$ is the transfer function of an AWG. $\mathcal{F}$ and $\mathcal{F}^{-1}$ 


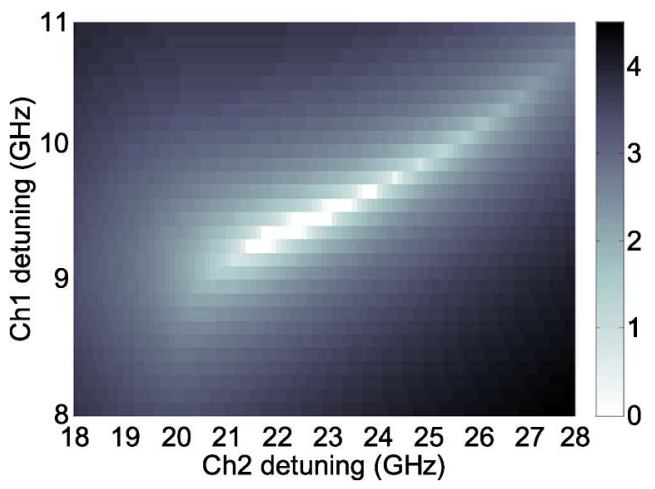

Fig. 1. Mandatory optical power attenuation in decibels for different wavelength detuning with respect to the FCC spectral mask. Ch1 and $\mathrm{Ch} 2$ are two independent AWG channels for incoherent superposition.

denote the Fourier and inverse Fourier transform notation, respectively. In our scheme, the Gaussian transfer function for each individual channel is assumed. Thus, the transfer function of the AWG is given by

$$
H_{\mathrm{AWG}}=\sum_{k} H_{G, k}(\omega)=\exp \left[-2 \ln (2)\left(\frac{\omega-\omega_{k}}{\Delta \omega_{3 \mathrm{~dB}, k}}\right)^{2}\right]
$$

where $k$ is the number of channels in consideration, and $\Delta \omega_{3 \mathrm{~dB}, k}$ is the 3-dB bandwidth of the $k$ th channel.

3) Simulation and Optimization: Numerical simulation is based on (2)-(4). A commercially available AWG with an ITU $50-\mathrm{GHz}$ spacing grid and $40-\mathrm{GHz} 3-\mathrm{dB}$ bandwidth is considered in our simulation. The simulation follows the process that two probe lights with identical continuous wave $(\mathrm{CW})$ optical power is used as the UWB optical carriers. The peak power of the pulsed pump light co-propagated with the probe light in the HNLF is then estimated in order to achieve $\pi$ phase shift on the both probe light. The time delay between the two asymmetric monocycle pulses are optimized to satisfy the pulse duration of the FCC-compliant UWB pulse designed in Section II-A. Finally, the wavelength detuning of the two probe lights relative to the two individual channels, respectively, are summarized in a 2-D map to estimate the compliance of the converted UWB pulse with the FCC spectral mask, as shown in Fig. 1. The overflow is defined as the minimum optical power required to be mandatorily attenuated to completely comply with the FCC spectral mask. According to this definition, the optimized wavelength detuning comes from where zero overflow is found. We will show in Section III that the designed FCC-compliant pulse matches very well with that measured in the experiment both in the time and frequency domains with the optimized wavelength detuning evaluated in simulation.

It is noted that, in a fully fledged UWB-over-fiber transmission system with a wireless link, the transmitted power, i.e., equivalent isotropic radiated power (EIRP), which considers the response of the UWB antenna, should comply with the FCC spectral mask instead. However, the wireless path is beyond the scope of this frame of study. As a result, the three data rates of 781.25 Mb/s, 1.5625 Gb/s, and 3.125 Gb/s under testing would rather be considered as the preliminary estimation of the potentially maximum speed of the system. The competence for a real transmission system could then be evaluated after including the wireless path with a multipath fading effect.

\section{EXPERIMENT AND RESULTS}

Our power-efficient UWB transceiver mainly consists of three building blocks, namely, generation and encoding, transmission, and receiver end. Air link is not considered in this stage.

\section{A. Generation and Encoding}

In Fig. 2, the experimental setup for FCC-compliant UWB pulse generation is shown. The principle and experimental setup are the same as we reported recently by employing the technique of multiple PM-IM conversions [21]. The inset of Fig. 2 shows the probe wavelengths located at different positions of the transfer function of the AWG channel led to the recovery of different polarities of asymmetric monocycles. In our case, channel 1 (191.4 THz) and channel 5 (191.6 THz) were used due to dense channel spacing of the AWG available. In any case, using adjacent channels should be avoided to reduce channel interference, especially for the detuning operation.

A digital communication analyzer (DCA), Agilent Infiniium DCA 86100A and an electrical spectrum analyzer (ESA), Agilent E4440A, with available bandwidth of $3 \mathrm{~Hz}$ to $26.5 \mathrm{GHz}$ were employed to measure the waveforms and spectra of the generated UWB pulses after two photodiodes (PDs), respectively. After optimizing the two probe wavelengths toward the designed spectrum and waveform we discussed in Section II, an FCC-compliant UWB spectrum and pulse shape had been experimentally achieved, as shown in Fig. 3(a) and (b), respectively. Every one per 125 bits with a $12.5-\mathrm{GHz}$ clock source resulting in an equivalent repetition rate of $100 \mathrm{MHz}$ was used for UWB pulse generation. A well-matched spectra and waveforms between theoretical design and experimental measurement were observed. The slight deviation of the spectral components in the low-frequency domain $(<1 \mathrm{GHz})$ was mainly due to the minor fluctuation of the UWB pulse tail originated from the pump pulse. However, it still scrupulously complied with the FCC spectral mask. The measured spectral efficiency within $3.1-10.6 \mathrm{GHz}$ was approximately $50.97 \%$. The probe light with optimized wavelengths of 1564.461 and $1566.223 \mathrm{~nm}$ were measured in the optical spectrum analyzer (OSA) before and after the AWG, as shown in Fig. 4(a) and (b), respectively. The Gaussian transfer functions of the two AWG channels plotted over the optical spectra of the two probe light further indicated the detuning of the two probe wavelengths, as shown in Fig. 4(a). Fig. 4(b) shown the optical spectra after channel filtering were asymmetric and the proportion of probe power changed as well.

\section{B. Transmission}

The transmission link consisted of a 22.5-km-long SMF and 4.4-km-long matched dispersion compensating fiber (DCF). In our scheme, the difference of the two probe wavelengths was approximately $2 \mathrm{~nm}$. As a result, the walk-off effect should be considered for long-distance transmission. In our experimental demonstration, a precise dispersion compensation for the operating wavelengths was carefully selected to prevent walk-off. 


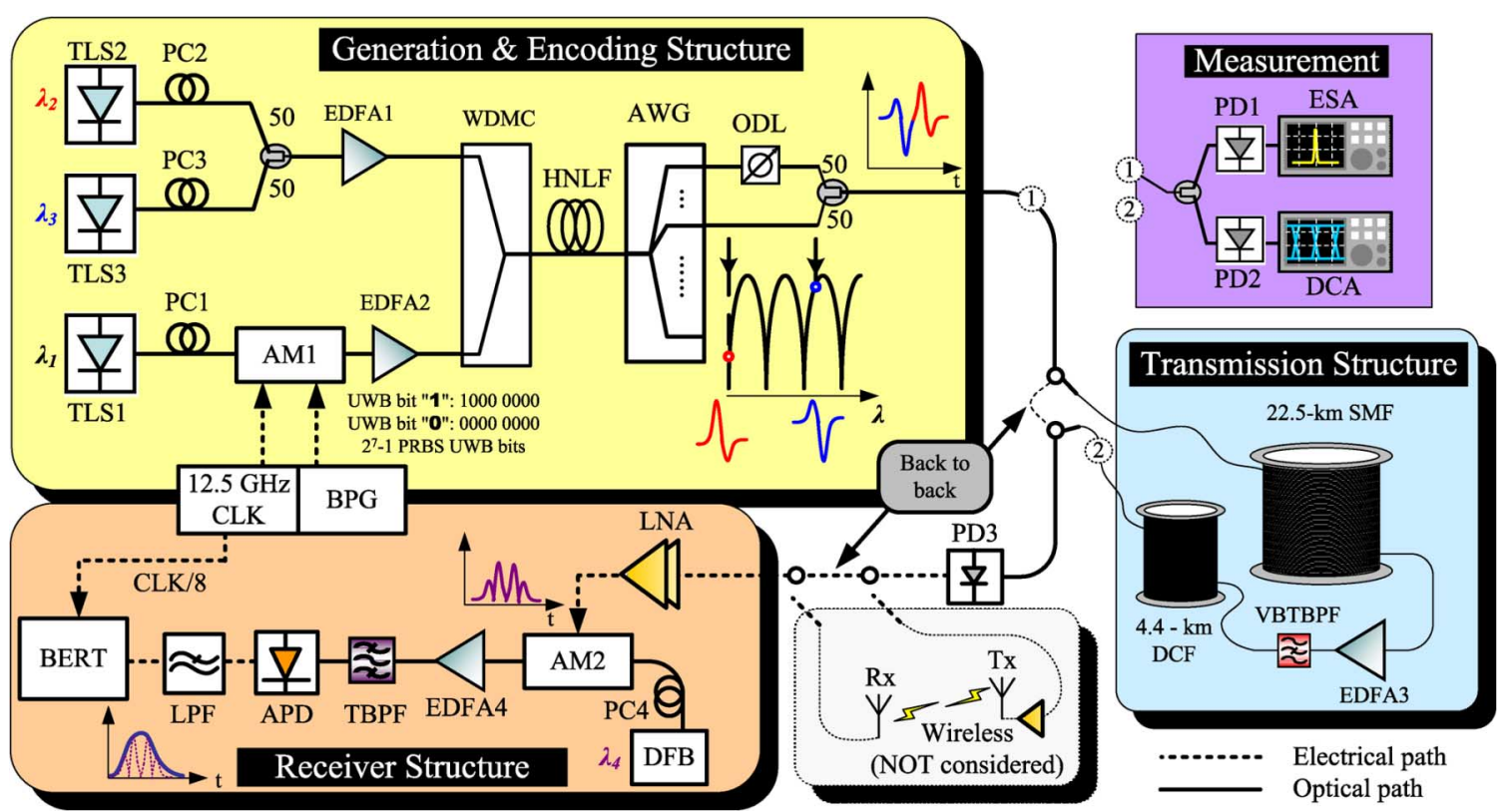

Fig. 2. Experimental setup of power-efficient UWB transceiver. Tunable laser source: TLS, amplitude modulator: AM, WDM coupler: WDMC, highly nonlinear fiber: HNLF, optical delay line: ODL, variable bandwidth tunable bandpass filter: VBTBPF, low-noise amplifier: LNA.

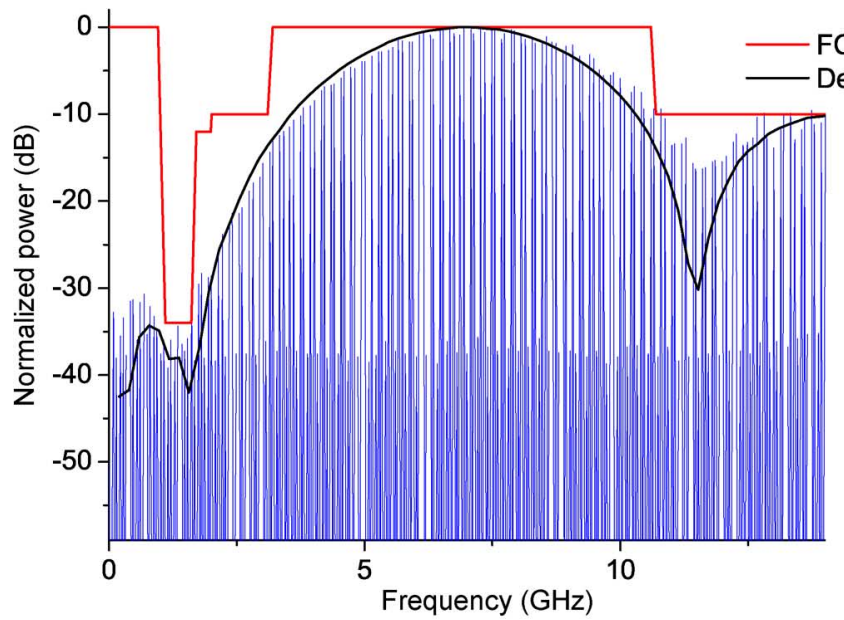

(a)

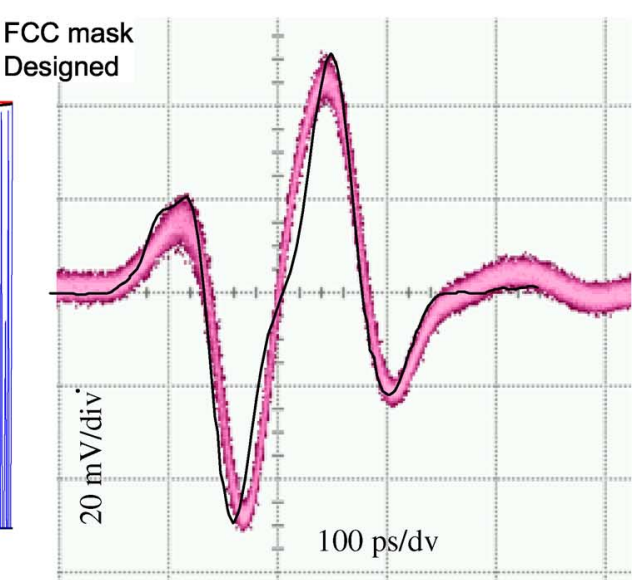

(b)

Fig. 3. Measured and designed: (a) electrical spectrum. (b) Corresponding FCC-compliant pulse.

In addition, the EDFA3 was used between the SMF and DCF to approximately compensate the $15-\mathrm{dB}$ fiber loss in the transmission link, which included the cascaded variable bandwidth tunable bandpass filter (VBTBPF) while setting the bandwidth to $2 \mathrm{~nm}$ to filter the two probe wavelengths.

It is noted that, if the wireless link with a pair of UWB antennas is also considered, the setup before the low-noise amplifier (LNA) should be also included in the transmission link. In that case, not only the response of the UWB antenna, but also the multipath fading effect, will further degrade the transmission performance as well. These extended issues, however, not discussed here, was still illustrated via a dotted-line box to show the system's integrity.

\section{Receiver}

Extensive reports focused on the generation of UWB pulses in recent years. However, only a few reports evaluated the per- formance of the whole UWB-over-fiber system. A digital signal processor (DSP) technology by bit-to-bit comparison is experimentally demonstrated in [15], [16], and [23]. This technique gives high sensitivity and is minimally dependent on signal-tonoise ratio (SNR). However, a very high-speed digital sampling oscilloscope (DSO) is required. By using an electrical local oscillator (LO), the UWB signal with central frequency around $5 \mathrm{GHz}$ is also down-converted to the baseband signal for direct measurement [24]. However, this technique obviously introduces a power penalty due to a broad UWB spectrum. The optical full-rectification technique used in [18] for UWB signal evaluation is attractive by using a pre-biased $\mathrm{LiNbO}_{3}$-based amplitude modulator (AM2) designed for $20 \mathrm{~Gb} / \mathrm{s}$, as shown in Fig. 2. It maintains the signal power for more precise evaluation compared with the half-rectification technique by using an electroabsorption modulator (EAM). In our scheme, we followed this technique and measured the power penalty for the 


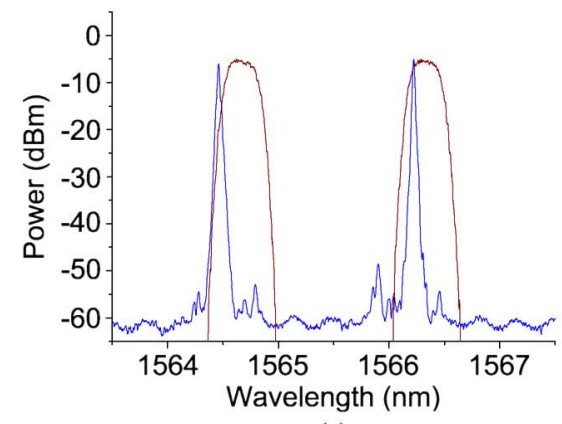

(a)

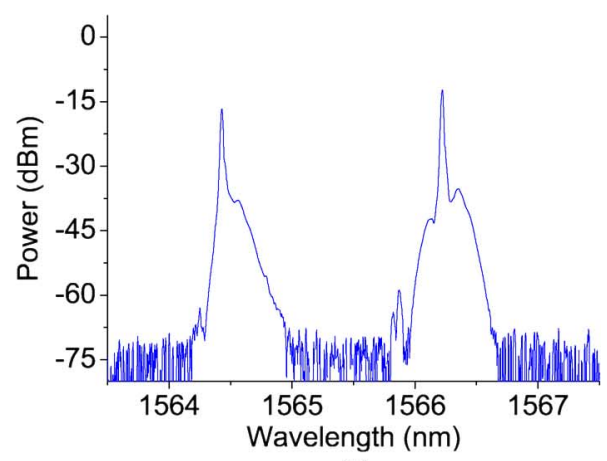

(b)

Fig. 4. Measured optical spectra of two probes: (a) before and (b) after AWG filtering with the transfer function of the corresponding channel of the AWG shown in (a).

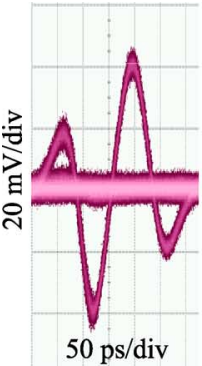

(a)

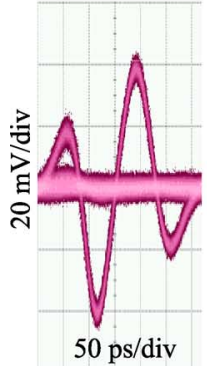

(b)

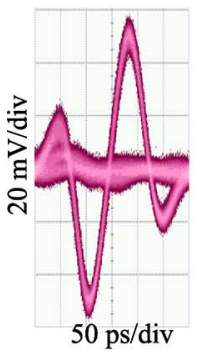

(c)

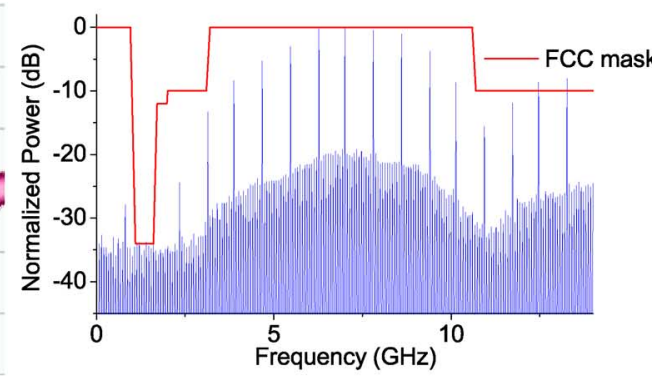

(d)

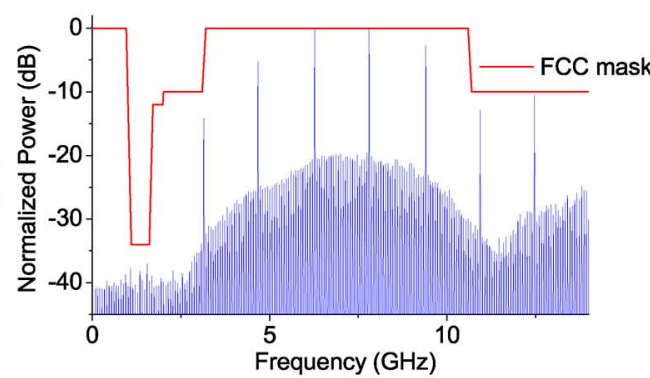

(e)

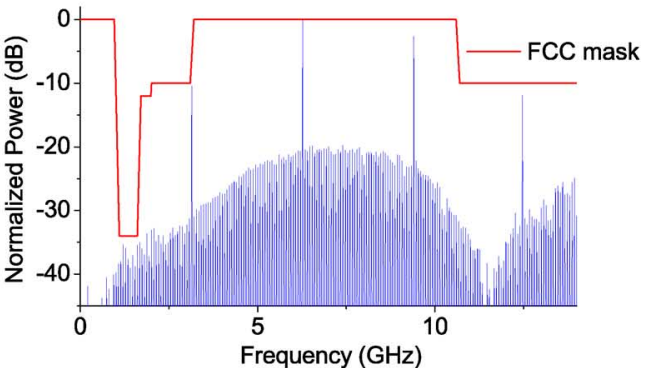

(f)
Fig. 5. Measured: (a)-(c) eye diagrams of FCC-compliant pulses. (d)-(f) Corresponding electrical spectra at $781.25 \mathrm{Mb} / \mathrm{s}, 1.5625 \mathrm{~Gb} / \mathrm{s}$, and $3.125 \mathrm{~Gb} / \mathrm{s}$, respectively, in back-to-back configurations.

previously mentioned fiber transmission link at the bit rates of $781.25 \mathrm{Mb} / \mathrm{s}, 1.5625 \mathrm{~Gb} / \mathrm{s}$, and $3.125 \mathrm{~Gb} / \mathrm{s}$.

In our receiver end, as shown in Fig. 2, the incoming UWB pulse was first detected by the PD3. The detected signal was then electrically amplified by an LNA with electrical bandwidth of $45 \mathrm{GHz}$ to maintain the pulse shape. The AM2 was pre-biased to shift the dc working point to the valley of the modulation function. As a result, a full rectification on the modulated optical signal emitting from a DFB laser at $1550 \mathrm{~nm}\left(\lambda_{4}\right.$, as shown in Fig. 2) was achieved. The EDFA4 after the AM2 was used to compensate the loss and maintain the optical power after the TBPF to be $3 \mathrm{dBm}$. An avalanche photodiode (APD) with bandwidth of $6.5 \mathrm{GHz}$ and a low-pass filter with 3-dB cutoff frequency of $3.74 \mathrm{GHz}$ were used to detect the fully rectified signal and performed as an integrating circuit simultaneously. The second BERT, Agilent 70842A, was synchronized by the clock source divided by $2^{n}(n=2,3,4)$ and used to evaluate the signal.

The FCC-compliant UWB pulse with a repetition rate of $100 \mathrm{MHz}$ measured in Fig. 3 proves that our generated UWB signal can be operated at any bit rate lower than the reciprocal of pulse duration of approximate 320 ps without power attenuation to respect the FCC spectral mask. As previously mentioned, benefiting from the ultrafast response of the HNLF, distortion-free UWB pulses can be generated. The UWB bit "1" consists of "1000 000000000000 " and UWB bit "0" consists of "0000 000000000000 ," resulting in an equivalent bit rate of $781.25 \mathrm{Mb} / \mathrm{s}$ (12.5 Gb/s divided by 16), following a pattern of $2^{7}-1$ PRBS were transmitted, while the corresponding pump power was increased to $11.09 \mathrm{dBm}$. Similarly, equivalent bit rates of $1.5625 \mathrm{~Gb} / \mathrm{s}(12.5 \mathrm{~Gb} / \mathrm{s}$ divided by 8$)$ and $3.125 \mathrm{~Gb} / \mathrm{s}$ $(12.5 \mathrm{~Gb} / \mathrm{s}$ divided by 4$)$ with each encoding bit length of 8 and 4 were used, respectively. The corresponding pump power was also increased to 14.55 and $18.32 \mathrm{dBm}$, respectively, to maintain the same modulation depth. The eye diagrams of generated pulses and the corresponding electrical spectra were immediately measured in back-to-back configurations, as shown in Fig. 5(a)-(f), respectively. It is obvious that the UWB signals transmitted at three different bit rates are all fully compliant with the FCC mask. The power level slightly above the FCC mask beyond $12 \mathrm{GHz}$ at $781.25 \mathrm{Mb} / \mathrm{s}$ will be eliminated by the UWB antenna. The eye diagrams of the signal transmitted at $781.25 \mathrm{Mb} / \mathrm{s}$ and $1.5625 \mathrm{~Gb} / \mathrm{s}$ are very clear. However, the eye diagram for the UWB signal at $3.125 \mathrm{~Gb} / \mathrm{s}$ is slightly overlapped due to minor fluctuations in the tail of the pulse previously mentioned, which will introduce additional power penalties in the receiver end. It will be explained in the following paragraphs.

The FCC-compliant UWB signals after 22.5-km SMF link transmission were measured both in the time and frequency domains, as shown in Fig. 6(a)-(c) and (d)-(f), respectively. It is 


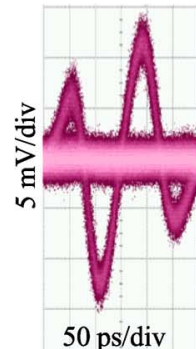

(a)

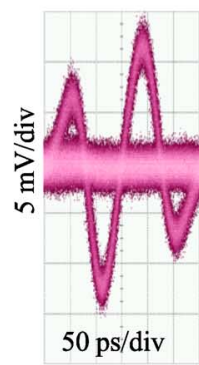

(b)

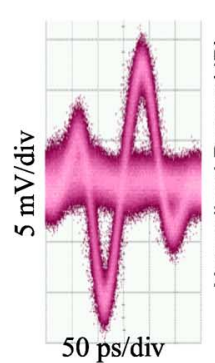

(c)

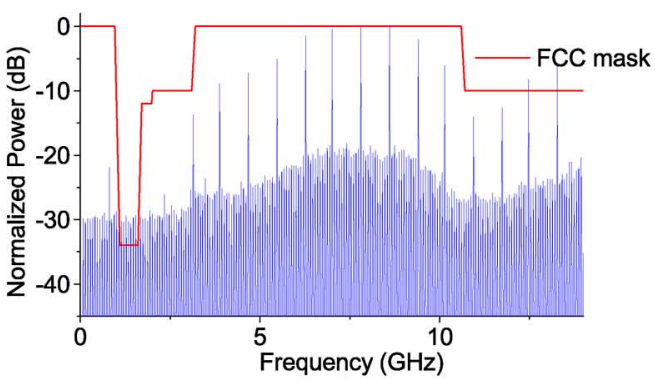

(d)

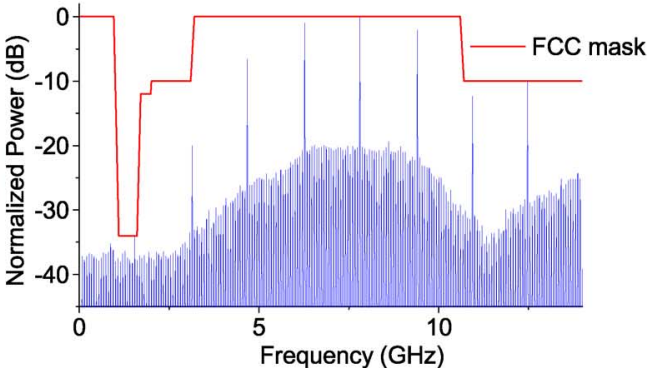

(e)

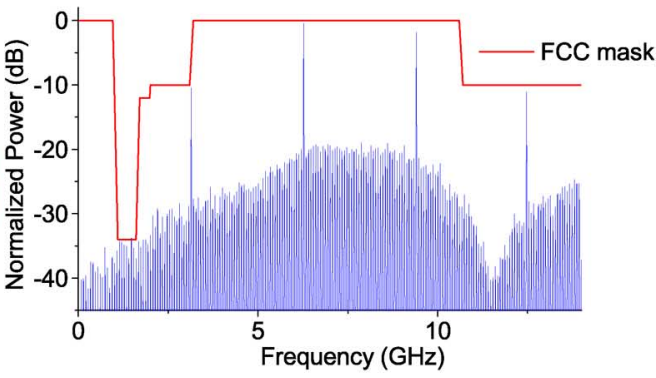

(f)
Fig. 6. Measured: (a)-(c) eye diagrams of FCC-compliant pulses. (d)-(f) Corresponding electrical spectra at $781.25 \mathrm{Mb} / \mathrm{s}, 1.5625 \mathrm{~Gb} / \mathrm{s}$, and $3.125 \mathrm{~Gb} / \mathrm{s}$ respectively after 22.5-km SMF link transmission.

noted that EDFA3 and VBTBPF are not used here. Compared with the results measured in Fig. 5(a)-(f), it is clear that the influence of the fiber transmission link on the UWB pulse shape and spectra is minimal. The electrical spectra after the fiber link transmission all fully comply with the FCC mask at three different speeds.

In the receiver end, the received UWB signals were used to intensity modulate an optical carrier emitted from a fourth DFB laser. The complex waveforms were measured by a wide bandwidth PD, HP 83440D with a bandwidth of $32 \mathrm{GHz}$, as shown in Fig. 7. It can be clearly identified that such a complex waveform is exactly the absolute amplitude deviated from the average level (dc part) of the received UWB signals. The fluctuation in the tail of the UWB pulse is also mitigated by AM2 due to the sinusoidal modulation function.

For a fair comparison of different bit rates, the power of probe wavelengths was maintained, as mentioned before, while the pump power was fixed at $15 \mathrm{dBm}$ at three different bit rates. In this case, the probe wavelengths were slightly regulated within $\pm 0.04 \mathrm{~nm}$ to maintain the spectra under the FCC mask. The eye diagrams after down-conversion to baseband signals for direct evaluation were shown in Fig. 8 without power attenuation before PD3. The left column of Fig. 8 stood for a back-toback configuration, while the right column represented after

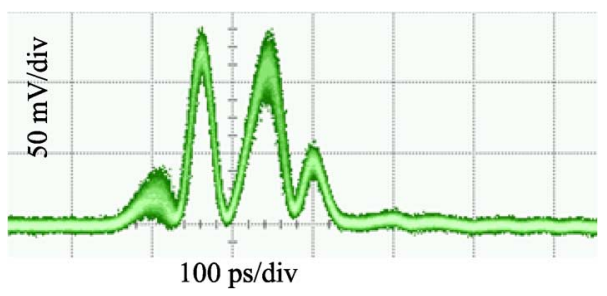

Fig. 7. Measured complex waveform after full rectification in the AM2.
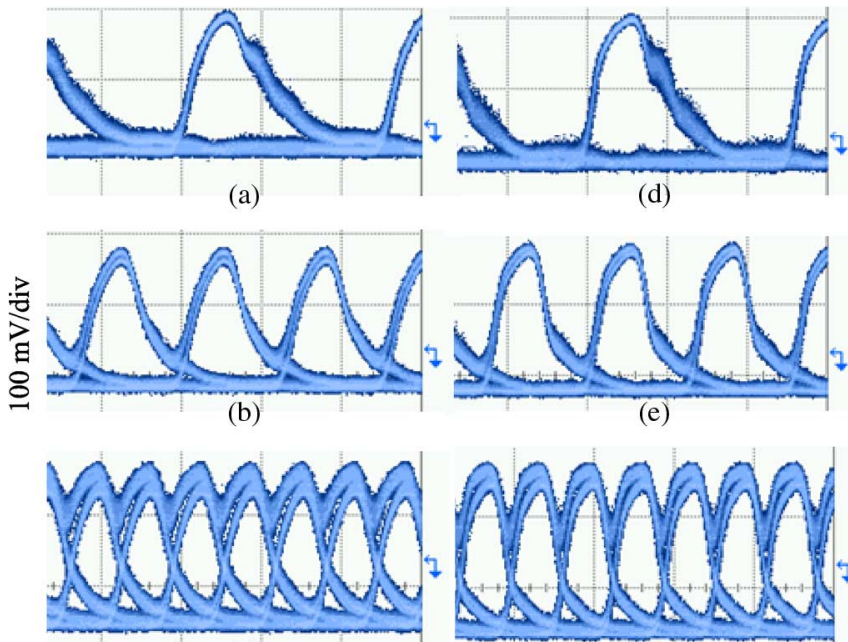

(c)
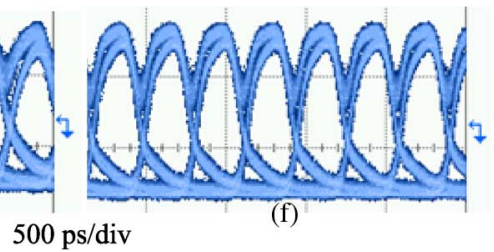

(f)

Fig. 8. Measured eye diagrams of down-converted baseband signals in front of the second BERT at error-free level in: (a)-(c) the back-to-back configuration and (d) and (e) after 22.5-km SMF link transmission at: (a) and (d) $781.25 \mathrm{Mb} / \mathrm{s}$, (b) and (e) $1.5625 \mathrm{~Gb} / \mathrm{s}$, and (c) and (f) $3.125 \mathrm{~Gb} / \mathrm{s}$, respectively.

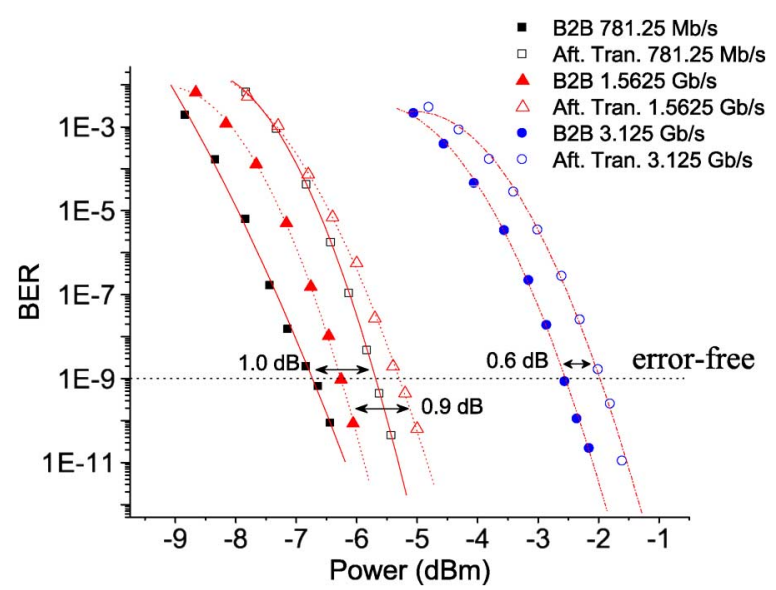

Fig. 9. BER measured for down-converted baseband signals at $781.25 \mathrm{Mb} / \mathrm{s}$, $1.5625 \mathrm{~Gb} / \mathrm{s}$, and $3.125 \mathrm{~Gb} / \mathrm{s}$ representing via the square, triangle, and circle in the back-to-back configuration (solid) and after 22.5-km SMF link transmission (unfilled), respectively.

22.5-km SMF link transmission. It was noted that with a clear eye opening, the converted signal with pulse duration long comparable with the bit period led to degradation in the BER. The unstable fluctuation in the tail of the pulse after conversion at $781.25 \mathrm{Mb} / \mathrm{s}$ was due to relatively wide bandwidth compared to the bit rate. A low-pass filter with an even lower 3-dB cutoff frequency would mitigate such instability.

The measured BER in the back-to-back configuration and after 22.5-km SMF link transmission is shown in Fig. 9. It is shown that the error-free level can be achieved when the 
received UWB optical signal power is $-6.74,-6.26$, and $-2.56 \mathrm{dBm}$ at $781.25 \mathrm{Mb} / \mathrm{s}, 1.5625 \mathrm{~Gb} / \mathrm{s}$, and $3.125 \mathrm{~Gb} / \mathrm{s}$ in the back-to-back configuration, respectively. The power penalties between $781.25 \mathrm{Mb} / \mathrm{s}$ and $1.5625 \mathrm{~Gb} / \mathrm{s}$ are mainly due to the different amplitudes of generated UWB pulses induced by the fixed pump power at the two bit rates. However, an even larger than $3-\mathrm{dB}$ power penalty is introduced at $3.125 \mathrm{~Gb} / \mathrm{s}$. Such a large power penalty is partly due to the above-mentioned reason, and is also partly due to the pulse overlapping observed in Figs. 5(c), 6(c), 8(c) and 8(f). However, the measured power penalties between the back-to-back configuration and after 22.5-km SMF link transmission for the three different bit rates are all less than $1 \mathrm{~dB}$ evaluated at the error-free level. The slightly curved trends of the measured BER beyond $1 \times 10^{-4}$ level are mainly due to the fact that the intensity applied to AM2 was distant from the linear working region of the modulator, and also due to the noise of the detector used.

\section{CONCLUSION AND Discussion}

We have theoretically investigated and experimentally demonstrated a simple and robust high-speed power-efficient UWB transceiver. The designed FCC-compliant pulse, which is fully compliant with the FCC spectral mask with spectral efficiency of $50.97 \%$, was experimentally demonstrated. The UWB signals transmitted at $781.25 \mathrm{Mb} / \mathrm{s}, 1.5625 \mathrm{~Gb} / \mathrm{s}$, and $3.125 \mathrm{~Gb} / \mathrm{s}$ following a pattern of $2^{7}-1$ PRBS can be error-free transmitted through a 22.5-km SMF and 4.4-km DCF fiber link with power penalties all less than $1 \mathrm{~dB}$, while maintaining their spectra to scrupulously respect the FCC spectral mask. Our scheme only consisted of an HNLF and a commercially available AWG, and without additional requirement of balanced receiver structure required by the spectral tailing technique [8], [18], [20]. Furthermore, a phase modulator (PM) would be used to replace the HNLF and pump pulse with even simple experimental setup and low cost if the PM could provide enough phase shift before overdriven when distortion occurs. Another issue of wavelength drifting of the two TLSs for optical carriers should be carefully controlled by introducing feedback circuits to eliminate influence from the environmental change in practical applications. However, it is not obviously observed in our experimental environment, and will be investigated in future.

\section{ACKNOWLEDGMENT}

The authors would like to thank Dr. Abtahi for very grateful discussion.

The authors would also like to acknowledge Sumitomo Electric Industries, Osaka, Japan, for providing the HNLF and Alnair Laboratories, Tokyo, Japan, for providing the VBTBPF.

\section{REFERENCES}

[1] "First report and order (revision of part 15 of the Commission's rules regarding ultra-wideband transmission systems)," FCC, Washington, DC, Apr. 2002.

[2] M. Abtahi, J. Magne, M. Mirshafiei, L. A. Rusch, and S. LaRochelle, "Generation of power-efficient FCC-compliant UWB waveforms using FBGs: Analysis and experiment," J. Lightw. Technol., vol. 26, no. 5, pp. 628-635, Mar. 2008.
[3] Y. L. Guennec, A. Pizzinat, S. Meyer, B. Charbonnier, P. Lombard, M. Lourdiane, B. Cabon, C. Algani, A. L. Billabert, M. Terre, C. Rumelhard, J. L. Polleux, H. Jacquinot, S. Bories, and C. Sillans, "Lowcost transparent radio-over-fiber system for in-building distribution of UWB signals," J. Lightw. Technol., vol. 27, no. 14, pp. 2649-2657, Jul. 2009.

[4] J. Yao, F. Zeng, and Q. Wang, "Photonic generation of ultrawideband signals," J. Lightw. Technol., vol. 25, no. 11, pp. 3219-3235, Nov. 2007.

[5] Q. Wang and J. Yao, "An electrically switchable optical ultrawideband pulse generator,' J. Lightw. Technol., vol. 25, no. 11, pp. 3626-3633, Nov. 2007.

[6] Y. Dai and J. Yao, "Optical generation of binary phase-coded direct-sequence UWB signals using a multichannel chirped fiber Bragg grating," J. Lightw. Technol., vol. 26, no. 15, pp. 2513-2520, Oct. 2008.

[7] V. Torres, K. Prince, and I. T. Monroy, "Ultrawideband pulse generation based on overshooting effect in gain-switched semiconductor laser,' IEEE Photon. Technol. Lett., vol. 20, no. 15, pp. 1299-1301, Aug. 2008

[8] C. Wang, F. Zeng, and J. Yao, "All-fiber ultrawideband pulse generation based on spectral shaping and dispersion-induced frequency-to-time conversion," IEEE Photon. Technol. Lett., vol. 19, no. 3, pp. 137-139, Feb. 2007.

[9] Q. Wang, F. Zeng, S. Blais, and J. Yao, "Optical ultrawideband monocycle pulse generation based on cross-gain modulation in a semiconductor optical amplifier," Opt. Lett., vol. 31, pp. 3083-3085, 2006.

[10] J. Dong, X. Zhang, J. Xu, D. Huang, S. Fu, and P. Shum, "Ultrawideband monocycle generation using cross-phase modulation in a semiconductor optical amplifier," Opt. Lett., vol. 32, pp. 1223-1225, 2007.

[11] H. Chen, M. Chen, T. Wang, M. Li, and S. Xie, "Methods for ultrawideband pulse generation based on optical cross-polarization modulation,” J. Lightw. Technol., vol. 26, no. 15, pp. 2492-2499, Aug. 2008.

[12] J. Li, B. P. P. Kuo, and K. K. Y. Wong, "Ultra-wideband pulse generation based on cross-gain modulation in fiber optical parametric amplifier," IEEE Photon. Technol. Lett., vol. 21, no. 4, pp. 212-214, Feb. 2009.

[13] J. Li, S. Fu, K. Xu, J. Wu, J. Lin, M. Tang, and P. Shum, "Photonic ultrawideband monocycle pulse generation using a single electro-optic modulator," Opt. Lett., vol. 33, pp. 288-290, 2008.

[14] E. Zhou, X. Yu, X. Zhang, W. Xue, Y. Yu, J. Mørk, and I. T. Monroy, "Photonic generation of ultrawideband monocycle and doublet pulses by using a semiconductor-optical-amplifier-based wavelength converter," Opt. Lett., vol. 4, pp. 1336-1338, 2009.

[15] X. Yu, T. B. Gibbon, and I. T. Monroy, "Experimental demonstration of all-optical 781.25-Mb/s binary phase-coded UWB signal generation and transmission," IEEE Photon. Technol. Lett., vol. 21, no. 17, pp. 1235-1237, Sep. 2009.

[16] R. Rodes, A. Caballero, X. Yu, T. B. Gibbon, J. B. Jensen, and I. T. Monroy, "A comparison of electrical and photonic pulse generation for IR-UWB on fiber links," IEEE Photon. Technol. Lett., vol. 22, no. 5, pp. 264-266, Mar. 2010.

[17] J. Li, Y. Liang, and K. K. Y. Wong, "Millimeter-wave UWB signal generation via frequency up-conversion using fiber optical parametric amplifier," IEEE Photon. Technol. Lett., vol. 21, no. 17, pp. 1172-1174, Sep. 2009.

[18] M. Abtahi, M. Mirshafiei, S. LaRochelle, and L. A. Rusch, “All-optical 500-Mb/s UWB transceiver: An experimental demonstration," $J$. Lightw. Technol., vol. 26, no. 15, pp. 2795-2802, Aug. 2008.

[19] M. Abtahi, M. Dastmalchi, S. LaRochelle, and L. A. Rusch, "Generation of arbitrary UWB waveforms by spectral pulse shaping and thermally controlled apodized FBGs," J. Lightw. Technol., vol. 27, no. 23, pp. 5276-5283, Dec. 2009.

[20] J. D. McKinney, I. S. Lin, and A. M. Weiner, "Shaping the power spectrum of ultra-wideband radio-frequency signals," IEEE Trans. Microw. Theory Tech., vol. 54, no. 12, pp. 4247-4255, Dec. 2006.

[21] E. Zhou, X. Xu, K. S. Lui, and K. K. Y. Wong, "A power-efficient ultra-wideband pulse generator based on multiple PM-IM conversions," IEEE Photon. Technol. Lett., vol. 22, no. 14, pp. 1063-1065, Jul. 2010.

[22] G. Agrawal, Nonlinear Fiber Optics, 4th ed. Burlington, MA: Academic, 2001.

[23] T. B. Gibbon, X. Yu, R. Gamatham, N. G. Gonzalez, and I. T. Monroy, "3.125 Gb/s impulse radio UWB over fiber transmission," in Proc. 35th Eur. Opt. Commun. Conf., Vienna, Austria, 2009, Art. ID P6.01.

[24] S. Pan and J. Yao, "A UWB over fibre transmitter reconfigurable for multiple modulation schemes," in Proc. 35th Eur. Opt. Commun. Conf., Vienna, Austria, 2009, Art. ID P6.04. 


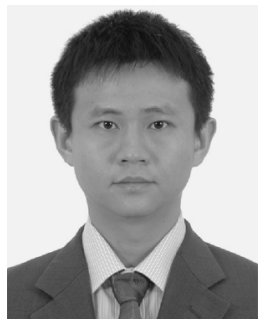

Enbo Zhou was born in December 1983. He received the B.Eng. degree in optoelectronics engineering from the Huazhong University of Science and Technology (HUST), Wuhan, China, in 2003, and the Ph.D degree in physical electronics from both HUST and Wuhan National Laboratory for Optoelectronics, Wuhan, China, in 2009.

In September 2003, he joined the Optical Fiber Device and Network Research Center, HUST, as a graduate student. From October 2007 to October 2008, he was with Department of Photonics Engineering, Technical University of Denmark, Lyngby, Denmark, as a Guest Researcher. $\mathrm{He}$ is currently with the Photonic Systems Research Laboratory, Department of Electrical and Electronic Engineering, University of Hong Kong, Hong Kong. His past research interests included ultrafast dynamics of SOAs and their applications. His current research interest includes microwave photonics.

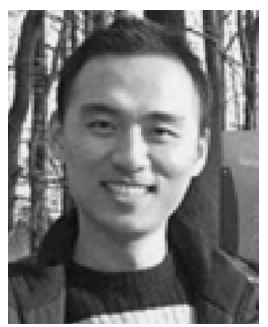

Xing Xu (S'09) received the B.Eng. degree in telecommunication engineering from Xidian University, Xi' an, China, in 2004, and is currently working toward the Ph.D. degree in electrical and electronic engineering at the University of Hong Kong, Hong Kong..

From 2004 to 2007, he was a Research and Development Engineer with the Fiberhome Telecommunication Company Ltd., Wuhan, China. During 2007 and 2008, he was a graduate student with the Delft University of Technology, Delft, The Netherlands. His main research interest is optical signal processing using optical parametric amplification and parametric wavelength exchange, optically powered networks, and microwave photonics.

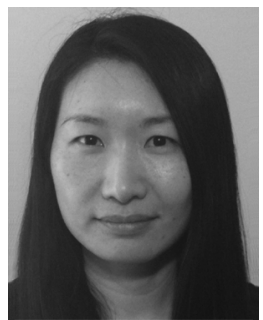

King-Shan Lui (S'00-M'03-SM'09) received the B.Eng. and M.Phil. degrees in computer science from the Hong Kong University of Science and Technology, Hong Kong, and the Ph.D. degree from the University of Illinois at Urbana-Champaign.

She then joined the Department of Electrical and Electronic Engineering, University of Hong Kong. Her research interests include network protocol design and analysis, optical networks, sensor networks, and quality-of-service issues.

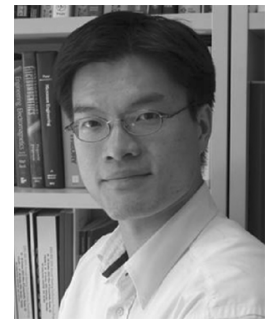

Kenneth Kin-Yip Wong (S'00-M'03) received the combined B.E. degree (first-class honors with a medal award) in electrical engineering and B.S. degree in physics from the University of Queensland, Brisbane, Qld., Australia, in 1997, and the M.S. and $\mathrm{Ph} . \mathrm{D}$. degrees from Stanford University, Stanford, CA, in 1998 and 2003, respectively, both in electrical engineering.

He was a member of the Photonics and Networking Research Laboratory, Stanford University. From 1998 to 1999, he was with Hewlett-Packard Laboratories, as a Research Engineer, during which time he contributed on projects that included parallel optics and vertical-cavity surface-emitting lasers (VCSELs). He was also an independent Consultant with Innovation CORE (a Sumitomo Electric Company), Santa Clara, CA, in 2004. He is currently an Assistant Professor with the Department of Electrical and Electronic Engineering, University of Hong Kong, Hong Kong. He has authored or coauthored over 50 journal and conference papers. He is the Reviewer for Optics Letters, the Journal of the Optical Society of America B, Optical Physics, Optics Express, IEE Electronics Letters, and Optics Communications. His past research interests included dense wavelength-division multiplexing (DWDM) systems, subcarrier multiplexed (SCM) optical systems, fiber nonlinearity, fiber optical parametric AMPs, and photonic crystal fibers.

Dr. Wong is a member of The International Society for Optical Engineers (SPIE) and the IEEE Lasers and Electro-Optic Society (LEOS). He is a reviewer for the IEEE PHOTONICS TECHNOLOGY LETTERS and the JOURNAL OF LightWAVE TECHNOLOGY. He was the recipient of the 2003 Optical Society of America (OSA) New Focus Student Award and the 2003 IEEE LEOS Graduate Student Fellowship. He was also the recipient of the 2005-2006 Best Teacher Award from the University of Hong Kong. 